Oikos 117: 1247-1257, 2008

doi: $10.1111 / j .2008 .0030-1299.16419 . x$,

(C) 2008 The Authors. Journal compilation (C) 2008 Oikos

Subject Editor: Tiffany Knight. Accepted 25 March 2008

\title{
When does ecosystem engineering cause invasion and species replacement?
}

\author{
Andrew Gonzalez, Amaury Lambert and Anthony Ricciardi \\ A. Gonzalez (andrew.gonzalez@mcgill.ca),Dept of Biology, McGill Univ., 1205 Docteur Penfield, Montreal, Quebec, H3A 1B1, Canada. - A. \\ Lambert, Laboratoire d'Ecologie et Evolution, Ecole Normale Supérieure, 46 rue d'Ulm, FR-75230 Paris Cedex 05, France. - A. Ricciardi, \\ Redpath Museum, McGill Univ., 859 Sherbrooke St. West, Montreal, Quebec, H3A 2K6, Canada.
}

\begin{abstract}
Introduced exotic species can dominate communities and replace native species that should be better adapted to their local environment, a paradox that is usually explained by the absence of natural enemies and by habitat alteration resulting from anthropogenic disturbance. Additionally, introduced species can enhance their invasion success and impact on native species by modifying selection pressures in their new environment through ecosystem engineering. We analyse a simple dynamic model of indirect competition for habitat between a non-engineering resident species and an engineering exotic species. The conditions for invasion and competitive exclusion of the resident by the exotic species and the range of dynamic outcomes suggested by the model are determined by the form of density dependence. We give simple criteria for the success of the invading species on dimensionless quantities involving rates of ecosystem engineering and of habitat degradation. The model's predictions offer an additional explanation for a range of invasion dynamics reported in the literature, including lag times between introduction and establishment. One intriguing result is that a series of failed invasions may successively reduce environmental resistance to subsequent invasion, through a cumulative effect of habitat transformation. More work is needed to determine the frequency and conditions in which engineering is required for successful establishment, and whether highly-successful (or high-impact) invaders are more likely to possess ecosystem engineering traits.
\end{abstract}

Biological invasions can transform ecosystems by altering their fundamental properties including energy flow, disturbance regimes, physical habitat structure, and biodiversity (D'Antonio and Vitousek 1992, Vitousek et al. 1997). Most introduced species fail to establish persistent populations, and many that become established have no discernible impact on the invaded ecosystem (Williamson 1996). Nevertheless, rates of invasion are increasing worldwide and most regions contain several hundreds to tens of thousands of invaders (Vitousek et al. 1997). Resistance to invaders posed by native species assemblages is generally weak, and invaders tend to have greater competitive effects on native species than vice versa (Vilá and Weiner 2004). A number of hypotheses have been developed to explain why many invaders dominate communities of native species that should be better adapted to their local environment. Among the explanations most commonly cited are the anthropogenic modifications of habitats to the direct and indirect benefit of the invader and the release of the invader from its natural enemies (Sax and Brown 2000, Keane and Crawley 2002, Colautti et al. 2004). An alternative, less explored explanation is that invaders perform an active role in their own success by modifying their new local environment. This process can occur both in ecological time through ecosystem engineering (Jones et al. 1997, Richardson et al.
2000, Cuddington et al. 2007) or evolutionary time through niche construction (Odling-Smee et al. 2003). Although both concepts share common conceptual ground, our approach does not incorporate evolutionary dynamics, thus we use the term 'ecosystem engineering' throughout.

Ecosystem engineering (Jones et al. 1997, Crooks 2002, Hastings et al. 2007) refers to the process by which organisms interact with and modify habitats, e.g. by emitting chemicals and nutrients, or by constructing artifacts such as nests, burrows, reefs, etc. Introduced engineers may generate habitat changes without a concomitant increase in their own fitness; e.g. the herbivorous activities of grass carp Ctenopharyngodon idella can reduce plant biomass without any apparent benefit from the resultant altered habitat structure, although other invading species may benefit (Pipalova 2002). However, they may also facilitate their own establishment and population expansion by reducing biotic resistance, i.e. interspecific interactions that limit the intrinsic growth rate of the invader. Examples involve a reduction of predation or competition (e.g. through allelopathy) that would otherwise limit invasion success (Keane and Crawley 2002), and the modification of local physical conditions (e.g. habitat structure, nutrient availability, microclimate, natural disturbance regimes) in favour of enhanced recruitment of the 
invader within the new habitat (Williamson 1996, Davis et al. 2000). Thus, native species that were well adapted to the previous environmental regime no longer have a competitive advantage.

Here we present theoretical and empirical evidence that ecosystem engineering may lead to the establishment and dominance of invaders. Cuddington and Hastings (2004) have considered the effects of ecosystem engineering on the rate and extent of invasion. Our study differs from these by explicitly considering the interaction (indirect interspecific competition) between two species, an exotic engineering species and a non-engineering resident. By extending a simple dynamical model (Gurney and Lawton 1996), we demonstrate that if the rate of ecosystem engineering is density-dependent then invasion may occur and may lead to the exclusion of a non-engineering resident species. We compare the dynamics of invasion given by linear and nonlinear density dependence and report a range of dynamical outcomes, including Allee effects which, combined with sequential invasion attempts, may explain the unpredictability of many invasions. We briefly review the empirical evidence for ecosystem engineering amongst exotic species, and provide specific evidence consistent with our model.

\section{The model}

We augmented the single-species ecosystem engineer model of Gurney and Lawton (1996) to include two species: a resident species and an exotic engineer. We assume that the exotic species depends upon a modified form of the local habitat which it engineers (either allogenically or autogenically, Jones et al. 1997) and which cannot be occupied by the resident species. We also assume that initial colonization requires either the presence of the engineered habitat or the arrival of individuals that possess sufficient energy reserves to begin engineering the local habitat. The model is deterministic and does not account for a possibly stochastic onset of colonization by the invasive species.

The invader (abundance I) can modify the local habitat (units of $\mathrm{H}_{1}$ ), used exclusively by the resident species

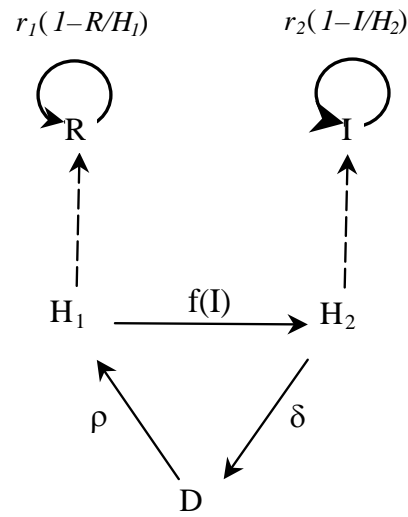

Figure 1. Representation of the model indicating the interrelations between the variables. Solid arrows represent the mapping from one state variable to another (e.g. $\mathrm{H}_{2}$ to $\mathrm{D}$ ), occurring at percapita rate indicated adjacent to the arrow (e.g. $\delta$ ); the dotted arrows represent resource dependence of the two species in the community. See Table 1 for full definition of the symbols used. (abundance R), to another form (units of $\mathrm{H}_{2}$ ) that meets its own ecological requirements and cannot be altered by the resident species.

Habitat quantity $\mathrm{H}_{\mathrm{i}}$ is expressed in units of carrying capacity for species $i$. The exotic species can transform the local habitat at a rate $f(I)$ per unit of habitat and per unit of time. The process is reversible; a unit of constructed habitat $\left(\mathrm{H}_{2}\right)$ decays at a constant rate $(\delta)$ into an intermediate degraded state (D) and a unit of degraded habitat returns at a constant rate $(\rho)$ to the original habitat $\left(\mathrm{H}_{1}\right)$, independently of the presence or absence of the two species. We assume that the total habitat stock $(\mathrm{T})$ remains constant so that the amount of degraded habitat is $\mathrm{T}-\mathrm{H}_{1}-\mathrm{H}_{2}$. Thus, the model (Fig. 1, Table 1) reads:

$$
\begin{aligned}
& \frac{\mathrm{dR}}{\mathrm{dt}}=\mathrm{r}_{1} \mathrm{R}\left(1-\frac{\mathrm{R}}{\mathrm{H}_{1}}\right) \\
& \frac{\mathrm{dI}}{\mathrm{dt}}=\mathrm{r}_{2} \mathrm{I}\left(1-\frac{\mathrm{I}}{\mathrm{H}_{2}}\right) \\
& \frac{\mathrm{dH}}{\mathrm{dt}}=\rho\left(\mathrm{T}-\mathrm{H}_{1}-\mathrm{H}_{2}\right)-\mathrm{f}(\mathrm{I}) \mathrm{H}_{1} \\
& \frac{\mathrm{dH}}{\mathrm{dt}}=\mathrm{f}(\mathrm{I}) \mathrm{H}_{1}-\delta \mathrm{H}_{2}
\end{aligned}
$$

where $r_{1}$ and $r_{2}$ are the intrinsic rates of increase for their respective populations. We present two cases in which the rate of habitat transformation $\mathrm{f}(\mathrm{I})$ of the exotic species is either a linear function bI or a non-linear function $\mathrm{cI}^{2}$ of its density. In the linear case, each invading individual transforms each unit of habitat used by the resident species at a constant rate (b) into that used by its conspecifics. In the non-linear case, habitat transformation is facilitative such that each invading individual transforms at a rate (cI) which is proportional to its population's abundance. The intrinsic rate of increase of the logistically growing populations sets the time-scale of the local dynamics.

Solving the equations, we obtain the following, where asterisks denote equilibrium values:

$\mathrm{R}^{*}=\mathrm{H}_{1}{ }^{*}$

$\mathrm{I}^{*}=\mathrm{H}_{2}{ }^{*}$

$\mathrm{f}\left(\mathrm{H}_{2}{ }^{*}\right) \mathrm{H}_{1}{ }^{*}=\delta \mathrm{H}_{2}{ }^{*}$

$\mathrm{f}\left(\mathrm{H}_{2}{ }^{*}\right) \mathrm{H}_{1}^{*}=\rho\left(\mathrm{T}-\mathrm{H}_{1}{ }^{*}-\mathrm{H}_{2}{ }^{*}\right)$

We note that the last three equations are sufficient to compute equilibrium values; we do not need to take into account the resident species' abundance $(R)$ because the last three equations define equilibrium without reference to it. In the following we are only concerned with the value at equilibrium of $\mathrm{H}_{1}, \mathrm{H}_{2}$ and I. In particular, the absence of invasion will be referred to as the trivial equilibrium $\left(\mathrm{H}_{1}\right.$, $\left.\mathrm{H}_{2}, \mathrm{I}\right)=(\mathrm{T}, 0,0)$, and all other outcomes will be referred to as invasion by the exotic species.

\section{The linear case $f(I)=b l$}

Figure 2 shows representative dynamics of this system when the resident $(\mathrm{R})$ starts at carrying capacity and the exotic engineering species (I) has very low initial density. Whether invasion occurs depends on the value of the per capita engineering rate (b) with respect to some threshold (equal 
Table 1. Parameters and their definition adopted in the model given in the text.

\begin{tabular}{|c|c|}
\hline Parameter & Definition \\
\hline $\mathrm{R}$ & abundance of resident species \\
\hline I & abundance of exotic engineering species \\
\hline $\mathrm{H}_{1}$ & quantity of native habitat \\
\hline $\mathrm{H}_{2}$ & quantity of engineered habitat \\
\hline $\mathrm{D}$ & quantity of degraded habitat \\
\hline$\delta$ & degradation rate of engineered habitat \\
\hline$\rho$ & regeneration rate of native habitat \\
\hline $\mathrm{T}$ & total habitat stock (both native and engineered) \\
\hline b & rate of individual engineering (linear model) \\
\hline C & rate of facilitative engineering (non-linear model) \\
\hline d & rescaled rate of degradation; $d=\delta / \rho$ \\
\hline $\mathrm{e}$ & $\begin{array}{l}\text { rescaled rate of engineering (non-linear model); } \\
\mathrm{e}=\mathrm{CT}^{2} / 2 \rho\end{array}$ \\
\hline
\end{tabular}

to $\delta / T$ ). If the engineering rate is below the threshold, invasion is impossible. If it is above the threshold, the equilibrium $\mathrm{H}_{2}{ }^{*}=\mathrm{I}^{*}=(\mathrm{T}-\delta / \mathrm{b}) /(1+\delta / \rho) ; \quad \mathrm{H}_{1}{ }^{*}=\delta / \mathrm{b}$, will be reached regardless of the (possibly low) initial abundance of the exotic species.

As $b$ is increased from 0 the range of dynamical outcomes of this model goes from invasion resistance to almost complete exclusion of the resident (Fig. 3). One can define the degree of exclusion as $1-\mathrm{H}_{1}{ }^{*} / \mathrm{T}=1-\delta / \mathrm{bT}$ which ranges from 0 when invasion is impossible to 1 , when the resident species is wiped out and replaced by the exotic engineer. Fixing a threshold, say 0.9 , for exclusion to be considered as complete, yields the following criterion for exclusion: $\delta / \mathrm{bT}<0.1$.

\section{The non-linear case $\mathrm{f}(\mathrm{I})=\mathrm{cl}^{2}$}

In the previous section, we showed a threshold value of the engineering rate (b) for persistence of the exotic species to occur, regardless of the initial conditions. Here, a simple criterion will also be given to assert whether persistence is possible, but invasion success will depend upon initial conditions (Fig. 4). In particular, in the region of parameter space where persistence is possible, there is a threshold value for the initial abundance of the exotic, below which invasion fails, and above which invasion succeeds. Another feature of this model is the existence of a bifurcation obtained by varying the rate of facilitative engineering $(c)$, habitat degradation $(\delta)$ or habitat regeneration $(\rho)$.

We will use the following two dimensionless quantities:

$$
\begin{aligned}
& \mathrm{d}=\delta / \rho, \\
& \mathrm{e}=\mathrm{cT}^{2} / 2 \rho
\end{aligned}
$$

where $\mathrm{d}$ is called the rescaled rate of habitat degradation and e the rescaled rate of engineering. (a)

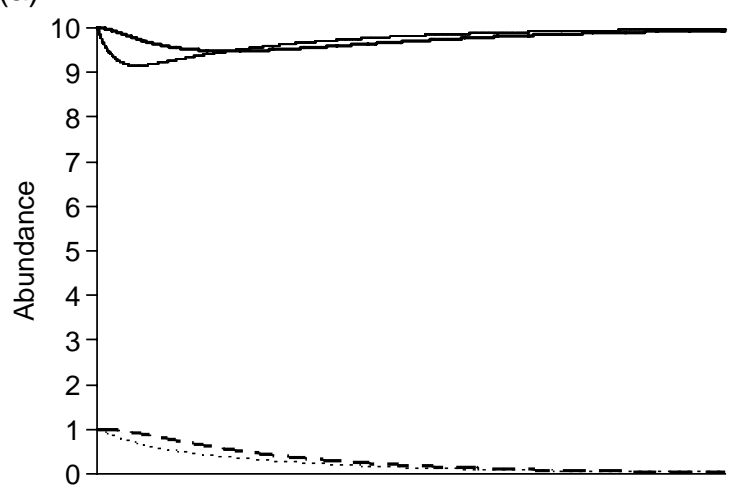

(c)

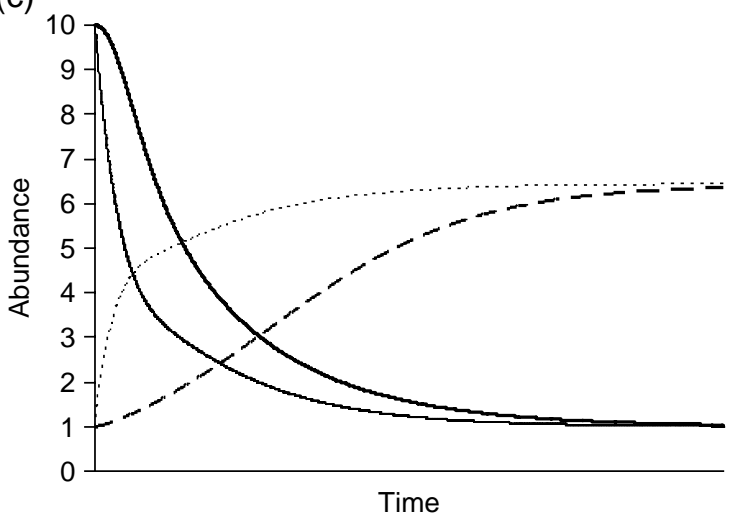

(b)

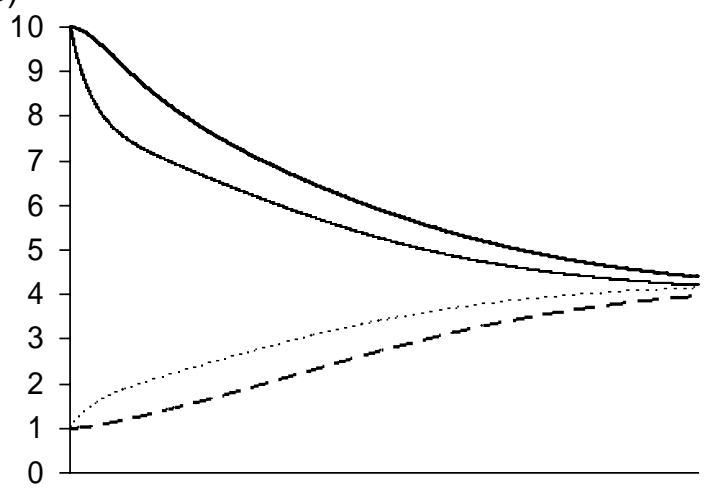

(d)

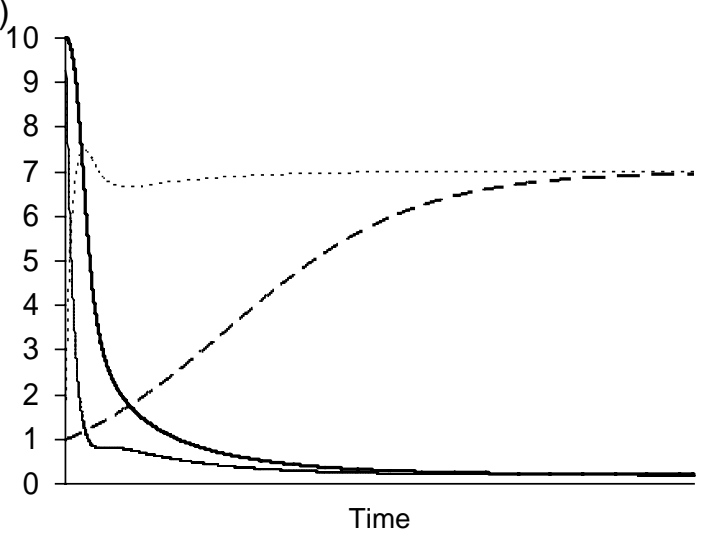

Figure 2. The effect of varying the habitat engineering rate (b) in the linear model. The population dynamics for four outcomes of the interaction between the resident $(R)$ and the exotic engineer (I) are shown, where: $r_{1}=r_{2}=1, \delta=2$. (a) unsuccessful invasion, $b=0.1$; (b) invasion and coexistence, $\mathrm{b}=0.5$; (c) invasion and dominance by the exotic engineer, $\mathrm{b}=2$; (d) invasion and exclusion of the resident, $\mathrm{b}=20$. Thick solid line $=$ resident species, thin solid line $=$ resident habitat, thick dotted line $=$ exotic species, thin dotted line $=$ engineered habitat. 


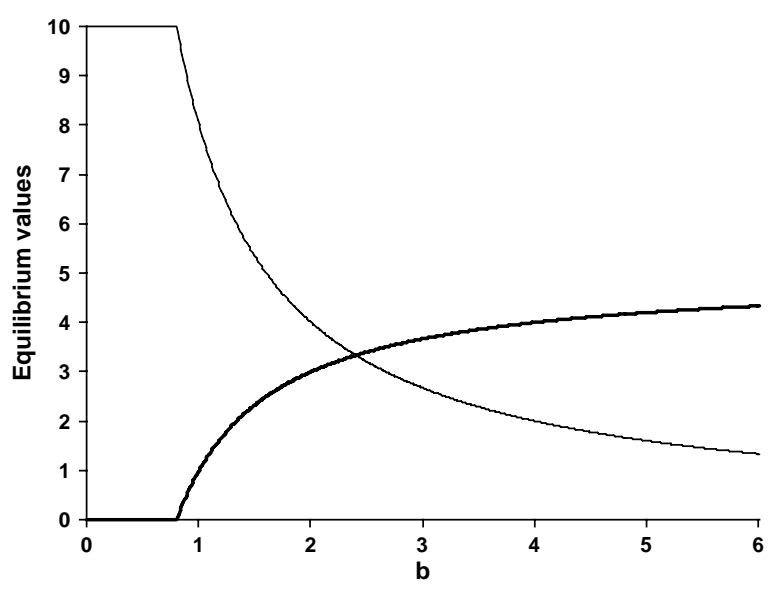

Figure 3. Equilibrium values of resident and exotic species as the habitat engineering rate (b) is varied in the linear model, where $\mathrm{T}=10, \delta=\rho=8$. Thick line $=$ exotic species $(\mathrm{I})$, thin line $=$ resident species (R). See Table 1 for symbols used.

\section{Determination of equilibria}

As in the linear model, invasion is strictly impossible if the rescaled engineering rate (e) is below some threshold (equal to $2 \mathrm{~d}(1+\mathrm{d})$ ). Otherwise, each species possesses two other equilibria aside from the trivial one (corresponding to zero invader abundance). The corresponding habitat abundances at equilibrium will be denoted by a capital letter for the largest and a lower case letter for the smallest. There are three possible equilibria: 1) the trivial equilibrium; 2) the unstable coexistence equilibrium denoted by $\left(\mathrm{H}_{1}, \mathrm{H}_{2}, \mathrm{I}\right)=$ $\left(\mathrm{H}_{1}{ }^{*}, \mathrm{~h}_{2}{ }^{*}, \mathrm{~h}_{2}{ }^{*}\right)$, and 3) the stable coexistence equilibrium denoted by $\left(\mathrm{H}_{1}, \mathrm{H}_{2}, \mathrm{I}\right)=\left(\mathrm{h}_{1}{ }^{*}, \mathrm{H}_{2}{ }^{*}, \mathrm{H}_{2}{ }^{*}\right)$. The trivial equilibrium is always stable. The unstable coexistence equilibrium is repulsive; if the initial abundance of the exotic species is smaller than some threshold depending on the initial values of $\mathrm{R}$ and $\mathrm{H}_{2}$, then it will suffer extinction, otherwise it will reach $\mathrm{H}_{2}{ }^{*}$. In some exceptional cases mentioned below, the only stable equilibrium is the trivial one (extinction of the exotic species).

We can get explicit expressions of the two nontrivial equilibria, and in particular, the degree of exclusion defined equal to $(1+a) / 2$, where

$\mathrm{a}=\sqrt{1-\frac{2 \mathrm{~d}(1+\mathrm{d})}{\mathrm{e}}}$

For example, the degree of exclusion is larger than 0.9 if a $>0.8$.

Let us consider the (rescaled) facilitative engineering rate (e) as the bifurcation parameter (Fig. 5a). When the value of the engineering rate (e) is smaller than the threshold $2 \mathrm{~d}$ $(1+d)$, invasion is impossible. As soon as this threshold is surpassed, the invader's abundance at equilibrium suddenly jumps upwards from 0 to $T / 2(1+\mathrm{d})$, and the resident's abundance at equilibrium jumps down from $T$ to $T / 2$. As the engineering rate (e) is further increased, this equilibrium splits into two equilibria, the stable equilibrium and the unstable equilibrium, along with an invasion threshold for the invader's initial abundance (allowing transformation of enough habitat to initiate growth). For initial conditions a)

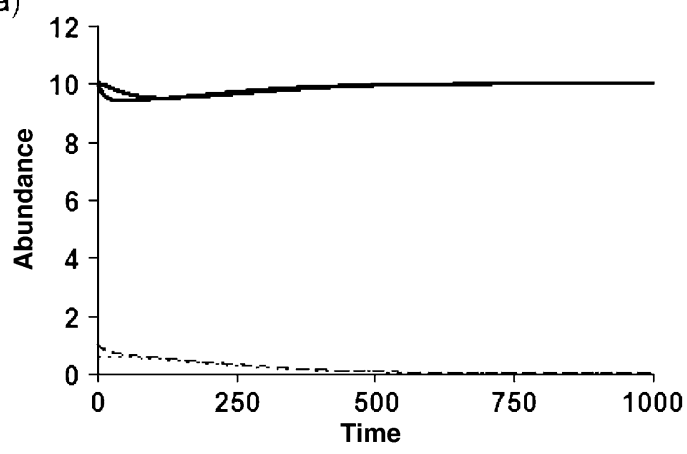

c)

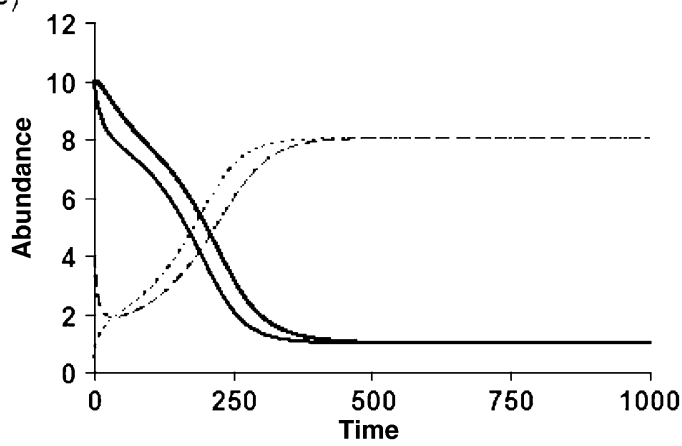

b)

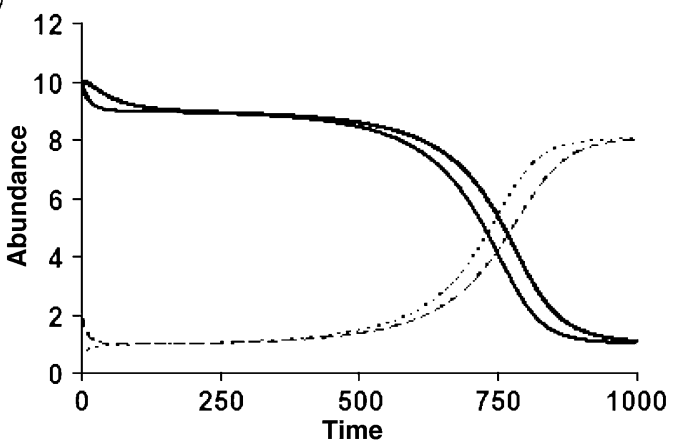

d)

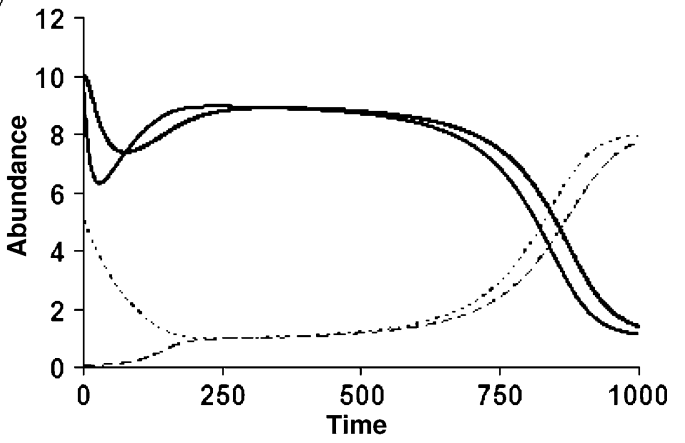

Figure 4. The effect of varying initial conditions in the non-linear model. Shown are the population dynamics for four outcomes of the interaction between the resident $(R)$ and the exotic engineer $(\mathrm{I})$, where $\mathrm{r}_{1}=1, \mathrm{r}_{2}=3, \delta=1, \rho=8, \mathrm{~T}=10, \mathrm{c}=0.125$. (a) $\mathrm{H}_{2}=0.5, \mathrm{I}=1$, no invasion, (b) $\mathrm{H}_{2}=0.5, \mathrm{I}=2$, delayed invasion, (c) $\mathrm{H}_{2}=0.5, \mathrm{I}=4$, rapid invasion, (d) $\mathrm{H}_{2}=5, \mathrm{I}=0.01$, two-step invasion. Thick solid line $=$ resident species, thin solid line $=$ resident habitat, thick dotted line $=$ exotic species, thin dotted line $=$ engineered habitat. 

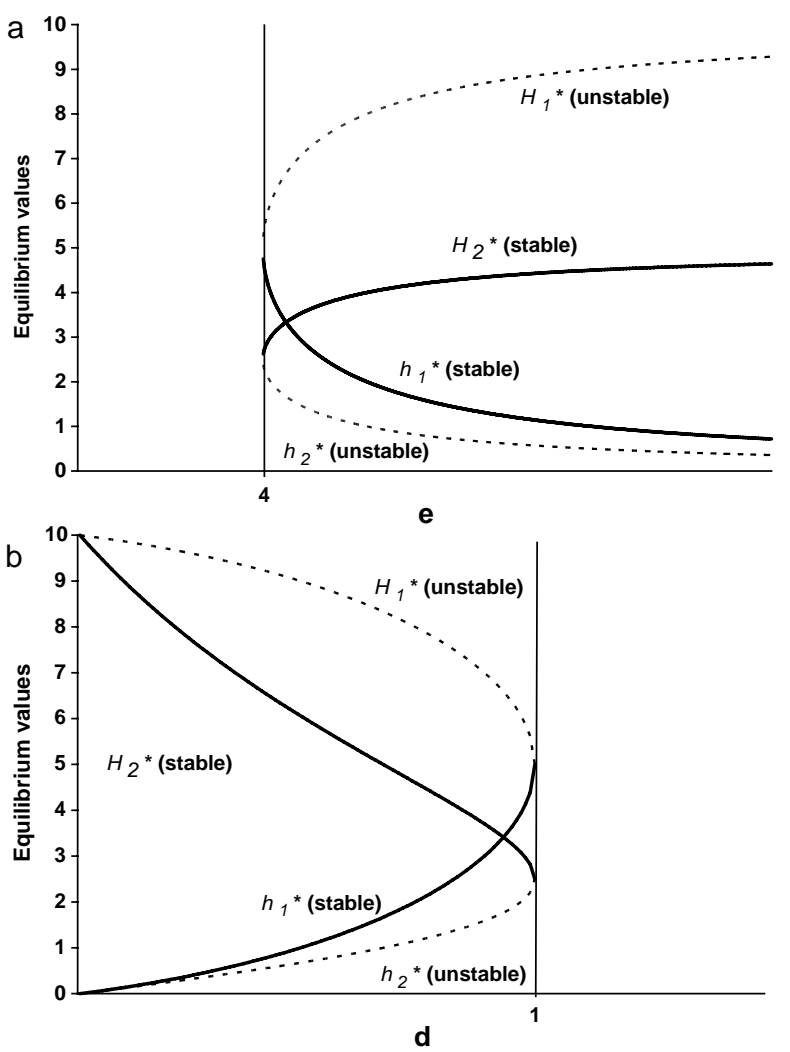

Figure 5. Bifurcation diagram for equilibrium values of resident and exotic species in the nonlinear model. (a) as $d$ (rescaled degradation rate of the transformed habitat) is varied $(e=4)$; $(b)$ as e (rescaled engineering rate) is varied $(\mathrm{d}=1)$; solid line $=$ stable equilibrium, dotted line $=$ unstable equilibrium. Trivial equilibrium is not shown.

where the resident species is at pre-invasion equilibrium (of the order of the habitat stock T), and the invading species comes in with a density lower than the invasion threshold, the exotic species goes extinct. If the invading species enters with an abundance greater than this threshold, it will reach the stable equilibrium $\mathrm{H}_{2}{ }^{*}>\mathrm{h}_{2}{ }^{*}$; and the abundance of the resident species will be reduced to $\mathrm{h}_{1}{ }^{*}\left(<\mathrm{H}_{1}{ }^{*}<\mathrm{T}\right)$ as shown in Fig. 5a. A similar bifurcation diagram can be drawn taking the rescaled habitat degradation rate (d) as the bifurcation parameter (Fig. 5b). It should be noted that it is not necessary to have $\mathrm{H}_{2}{ }^{*}>\mathrm{h}_{1}{ }^{*}$ at (stable) equilibrium. In other words, the engineering species may invade but not necessarily exclude the resident species (coexistence).

Figure 6 provides an overview of the dynamics obtained in three main regions of the parameter space defined by the rate of habitat transformation (e) and habitat degradation (d). In the region of possible invasion whether invasion occurs depends upon the initial abundance of the exotic engineer: below the invasion threshold extinction of exotic is assured (Fig. 6a) whilst an initial abundance above it triggers invasion (Fig. 6b). Figure 6e shows the region of invasion failure regardless of initial abundance and the rate of engineering and habitat degradation. There is also a narrow region where coexistence is possible (Fig. 6e) but if $r_{2}$ is sufficiently large, and given the additional condition
$2 \mathrm{~d}(1+\mathrm{d})<\mathrm{e}<\frac{\left(\mathrm{d}^{2}+\mathrm{d}-1\right)^{2}}{2(\mathrm{~d}-1)}$

the coexistence equilibrium is unstable. Specifically, there still remain three equilibria, but only the trivial one is stable. The consequence is that any attempt to invade is certain of extinction, regardless of the initial abundance. As seen on Fig. 6d, the parameter region is relatively narrow, and implies in particular that $\mathrm{d}>\mathrm{d}_{0}=(1+\sqrt{ } 5) / 2 \approx 1.62$ and $\mathrm{e}>\mathrm{e}_{0}=4+2 \sqrt{ } 5 \approx 8.47$. This deleterious effect (impossible invasion) of large values of $r_{2}$ can result in overcompensating growth; if the exotic species increases too rapidly, the pressure of habitat transformation causes an abrupt decrease of habitat 1 , which in turn triggers the extinction of the exotic species.

\section{Discussion}

\section{Summary of model predictions}

\section{Invasion success and competitive exclusion}

We demonstrated that for an exotic engineer invasion success depends on the rate of engineering (b or $c$ ), the habitat degradation rate $\delta$, the habitat recovery rate $\rho$, and the total habitat stock $T$. This can be seen on the bifurcation diagrams of Fig. 3 and 5. In the case when the per capita rate of habitat engineering is constant (b), invasion is possible as soon as $\mathrm{b}$ is above a certain threshold value (equal to $\delta / T$ ). In the non-linear case when the rate of habitat engineering is proportional to the invader's abundance (c), invasion occurs as soon as e $>2 \mathrm{~d}(1+\mathrm{d})$; where we defined the rescaled engineering rate $\mathrm{e}=\mathrm{cT}^{2} / 2 \rho$ and the rescaled degradation rate $d=\delta / \rho$. These conditions define successful invasion but not necessarily exclusion of the resident and hence species replacement. In the linear case the resident species is excluded when $\delta / \mathrm{bT}<0.1$, and in the non-linear case when $\mathrm{d}(1+\mathrm{d}) / \mathrm{e}<0.18$.

It is worth noting that the criterion for invasion of the exotic engineer depends solely on dimensionless quantities $-\delta / \mathrm{bT}$ in the linear case, $\mathrm{d}$ and e in the non-linear case these summary parameters allowed us to test the joint effect of the different parameters on the outcome of the dynamics.

\section{Invasion dynamics}

In the region of parameter space where persistence of the invader is possible, there is a major difference between the linear and non-linear models. In the linear case, as soon as persistence is possible, it will occur regardless of the initial invader's abundance. In the non-linear case, invasion is possible when the invader (I) and its habitat $\mathrm{H}_{2}$ are above some threshold. This is an Allee effect (Taylor and Hastings 2005) that emerges from the assumption that habitat engineering is facilitative, so that low abundances of the invading engineer are insufficient to prevent population extinction. Allee effects can arise by several processes (e.g. predator avoidance, sexual mating) in invasion biology but our model suggests that ecosystem engineering may be an additional process that merits further study.

Another prediction that arises from the engineering perspective taken here is that the persistence threshold for the invader actually depends negatively upon the initial 


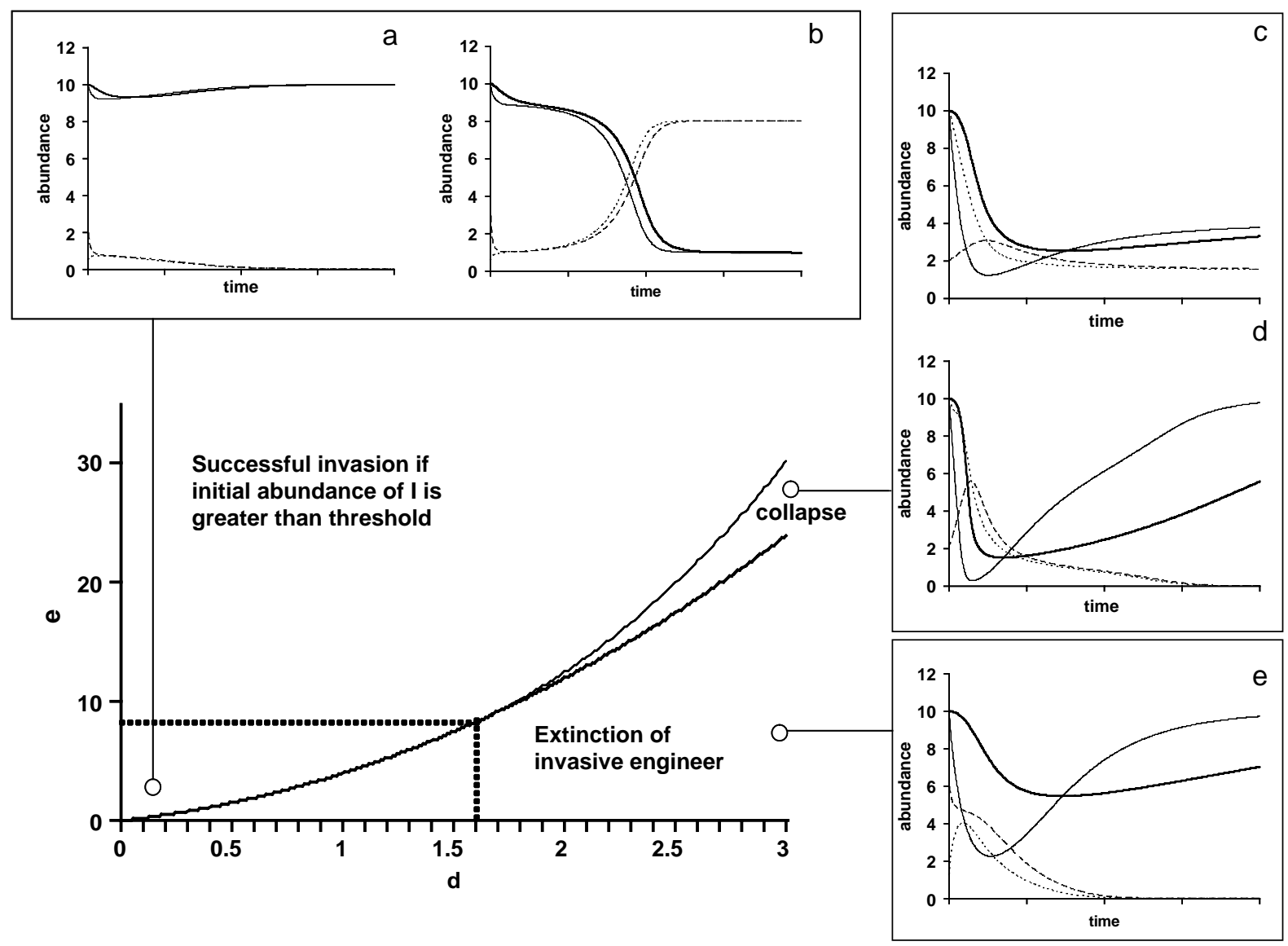

Figure 6. Nonlinear case - outcome of the dynamics in the parameter space (d,e). The narrow region of 'collapse' indicates a parameter region, $\left(\mathrm{d}_{0}, \mathrm{e}_{0}\right)(1.62,8.47)$, for which the persistence of the invasive species is impossible, despite the existence of two non-trivial unstable equilibria, provided $r_{2}$ is large enough with respect to $r_{1}$. The graphs show typical population dynamics: (a) region of possible invasion but initial abundance of the invader is below the persistence threshold resulting in the extinction of the invader, $\delta=1, \rho=8$, $c=0.125, T=10$, (b) the same region, same parameter values, but the initial abundance of the invader is above the persistence threshold triggering invasion, (c) region of collapse, but the invader's growth rate $r_{2}$ is sufficiently small, allowing coexistence, $\delta=15, \rho=5, c=$ $2.5, T=10, r_{2}=5$, (d) same region and the same parameter values as the preceding case but with large $r_{2}$ now the invader is driven to extinction by overexploitation of $\mathrm{H}_{1}, \mathrm{r}_{2}=20$, (e) this is the region of invasion failure regardless of initial abundance, $\delta=12, \rho=3, \mathrm{c}=$ $0.5, \mathrm{~T}=10$. Thick solid line $=$ resident species, thin solid line $=$ resident habitat, thick dotted line $=$ exotic species, thin dotted line $=$ engineered habitat.

stock of engineered habitat $\mathrm{H}_{2}$. A succession of failed attempts of invasion with each causing the accumulation of some transformed habitat (assuming the habitat degradation rate is slower than the engineering rate) will thus decrease the value of the persistence threshold for subsequent invasion attempts, and thus environmental resistance to invasion will decline over time. Invasion may occur after a series of failed attempts, not because the propagule size has finally overcome a fixed persistence threshold, but because this threshold has been lowered by the presence of transformed habitat due to the preceding invasion attempts (a legacy effect).

\section{Invasion waiting time and transient equilibria}

Another prediction of the non-linear model is the dependence of the waiting time to invasion upon the initial conditions. In Fig. 4, the parameters of the model are kept constant and belong to the region of the parameter space where persistence is possible, but the initial conditions are varied: a low initial abundance (i.e. a small introduced propagules size) leads to a failed invasion (Fig. 4a); when the initial abundance is near the threshold, a long lag time may precede invasion (Fig. 4b); and above the threshold promotes rapid invasion (Fig. 4c). In the case where the invader's initial abundance is low but an intermediate amount of transformed habitat is available, invasion occurs in two steps: the initial rapid growth of the invader reaches a transient equilibrium slightly above threshold, followed by a lag time before actual invasion (Fig. 4d).

An interesting feature of the non-linear model is the existence of a discontinuity of equilibrium abundances at a critical point. Recall that in the linear case, as the engineering rate $\mathrm{b}$ increases beyond the threshold $\delta / T$, the invader's abundance at equilibrium increases continuously from zero and the resident's abundance decreases continuously from $\mathrm{T}$ (Fig. 3). In contrast, in the non-linear case, a slight increase of the value of the (rescaled) facilitative engineering rate e near its critical value $2 \mathrm{~d}(1+\mathrm{d})$ results in a jump of the equilibrium abundances (Fig. 5a): the equilibrium value of the resident species jumps from total 
occupation (and zero invader individuals) to half-occupation (and near half-occupation by the exotic species).

\section{Invasion collapse}

Lastly, it is worth noting the importance of the maximum growth rate $r_{2}$ of the exotic species in the non-linear model (Fig. 6d). For a narrow region of the parameter space, the outcome of the dynamics will switch from possible invasion (if $r_{2}$ is small) to collapse of the exotic species through overexploitation of the resident habitat (if $r_{2}$ is large).

\section{Empirical evidence for the role of ecosystem engineering in invasion biology}

The most popular explanation for the exceptional success and impact of an invader is its escape from natural enemies that can limit its abundance (Keane and Crawley 2002, Colautti et al. 2004). Ecosystem engineering is an additional mechanism by which an invader can dominate native species that are expected to be better adapted to the local environment. By altering habitats invaders can affect the fluxes of resources in ecosystems in a variety of ways (Mack and D'Antonio 1998, Crooks 2002, Bais et al. 2003). It is not surprising that ecosystem engineering modifies selection pressures not only on the engineer and its descendants but also on other species that share the local environment. However, the effects of ecosystem engineering vary markedly among taxa; they tend to be generally weak in highly mobile species such as introduced birds and fishes, but strong in sedentary species such as bivalves and plants (Mack and D'Antonio 1998, Crooks 2002, Gutiérrez et al. 2003). Some exotic plants and animals produce rapid changes in disturbance regimes that promote their dominance over native species (Mack and D'Antonio 1998, Brooks et al. 2004). In terrestrial environments, invasive grasses such as European cheatgrass Bromus tectorum can change the fuel properties and ultimately the frequency and intensity of fire in their new environment. The high surface area to volume ratio of their leaves, the rapid accumulation of leaf litter, and the microclimate created by these grasses favor increased frequency of wildfires. Cheatgrass recovers rapidly following a wildfire, pre-empts the growth of competing native shrubs, and initiates a positive feedback that leads to dominance by the invader (D'Antonio and Vitousek 1992, Brooks et al. 2004, Link et al. 2006). The risk and intensity of fire increase asymptotically with the percent cover of cheatgrass (Fig. 7a).

Exotic plants also alter soil chemistry and soil biota in ways that directly favour the invader and that are detrimental to resident species (Talley and Levin 2001, Kourtev et al. 2002, Bais et al. 2003, Duda et al. 2003, Callaway et al. 2004). Exotic aquatic plants may similarly alter water quality. The dense cover created by water hyacinth Eichornia crassipes, an invasive floating plant, reduces atmospheric oxygen exchange with surface waters and causes an accumulation of decaying vegetation on bottom sediments - thereby producing anoxic conditions that promote an increased release of phosphate, which stimulates further plant growth (Ntiba et al. 2001). Moreover, water hyacinth exudes chemicals that have allelopathic effects on phytoplankton, its competitors for nutrients
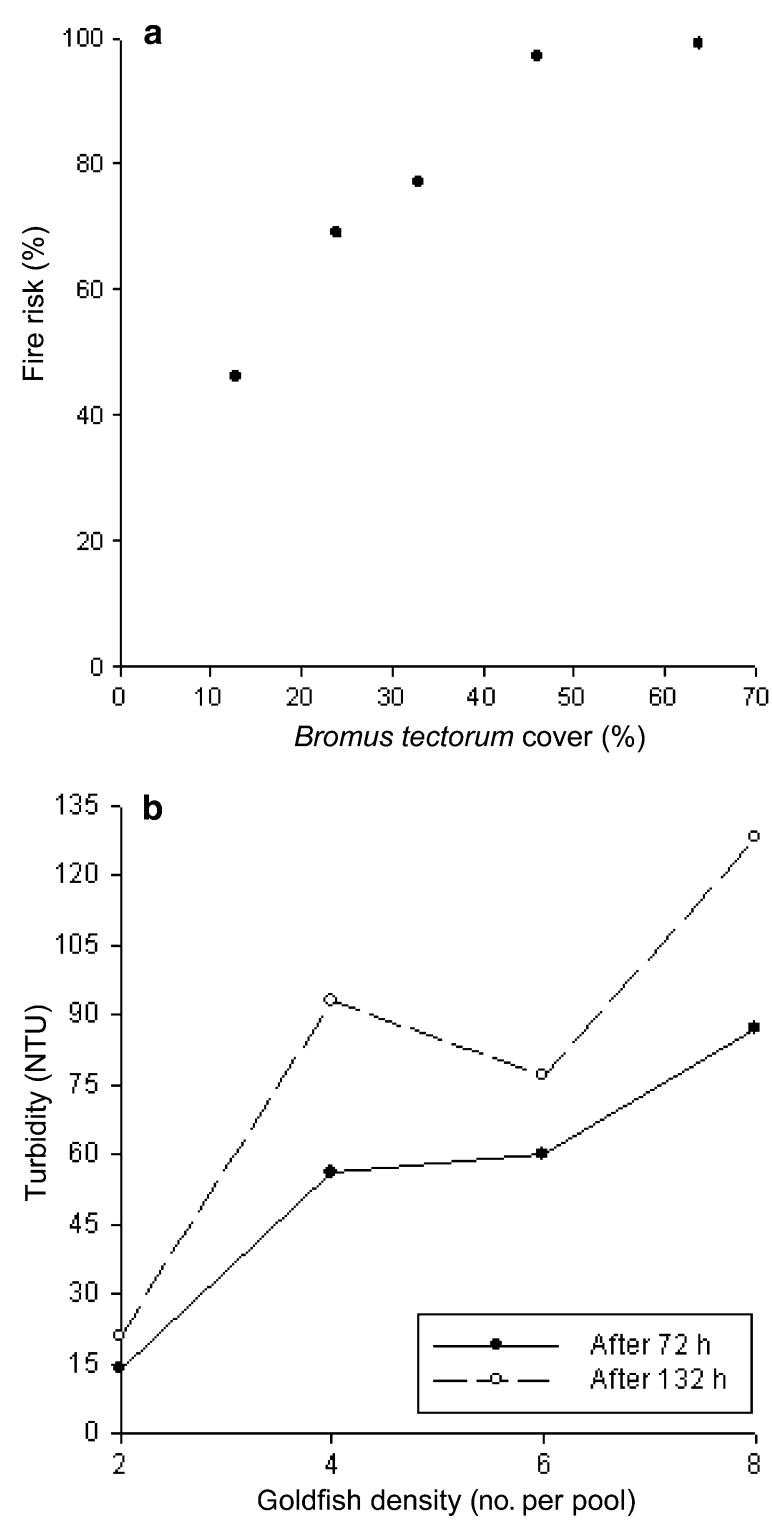

Figure 7. Empirical examples linking density to ecosystem engineering. (a) fire risk increases with the cover of an invasive grass ( $B$. tectorum), which thrives in the absence of native competitors removed by fire. Redrawn from Link et al. (2006). (b) effect of increasing goldfish abundance on turbidity in experimental pools (data from Richardson et al. 1992). Turbidity reduces the effectiveness of visual predators and competitors of goldfish.

(Yang et al. 1992). These activities can contribute to a shift in plant dominance in lakes (Scheffer et al. 2003).

Although ecosystem engineering appears to be common among species (Jones et al. 1997, Odling-Smee et al. 2003), we expect that the recipient community will be subject to greater negative effects from exotic ecosystem engineers, whose activities are likely to be novel - and therefore native species are less likely to be adapted to the resultant environmental changes. This resonates with the prediction by Odling-Smee and colleagues (2003) that if an invader replaces a resident species that performs different engineering activities, it is likely to alter the ecosystem to the detriment of other resident species. We focus now on 
specific support we have found in the literature for the model presented above.

\section{Empirical evidence for the linear model}

There are documented examples illustrating each of the linear model outcomes of the interaction between exotic engineers and resident species. Coexistence (Fig. 2b) is likely the most common outcome in nature, as most invasions do not cause extinctions (Gido and Brown 1999, Sax et al. 2002). They may nonetheless cause a population reduction in previously dominant resident species (Fig. 2c), as occurred after the invasion of the Rhine River by the mud amphipod Corophium curvispinum, a burrowing crustacean that requires fine sediment with which to create tubicolous galleries for shelter and to facilitate filter-feeding. Amphipods gather and consolidate mud to cover rock surfaces, an engineering activity that inhibits resident invertebrate species adapted to hard substrates. In the Rhine River, the deposition of mud increased, while the density of other invertebrates adapted to hard substrate (zebra mussels, hydropsychid caddisflies, gammarid amphipods) declined with amphipod density; zebra mussels, which had a density of $\sim 50000 \mathrm{~m}^{-2}$, declined by three orders of magnitude as adults became fouled and larval settlement was inhibited by mud deposits (van den Brink et al. 1993, van der Velde et al. 1994).

The density-dependent effects of an invasive engineer may also result in the exclusion of resident species (Fig. 2d), as demonstrated by the introduction of goldfish and carp to shallow lakes. The benthic feeding activities of these fishes disturb the sediment and increase turbidity (Fig. 7b), thereby occluding fish coloration and reducing risk of predation, while simultaneously causing severe reductions in rooted plants; the physical changes and associated impacts on plants are linearly correlated with the invader's abundance (Richardson and Whoriskey 1992, Richardson et al. 1995, Richardson 1996, Lougheed et al. 1998, Zambrano et al. 1999).

\section{Empirical evidence for the non-linear case}

One likely example of the non-linear form of facilitative ecosystem engineering is the colonization by introduced zebra mussels Dreissena polymorpha of areas where the availability of preferred substrates is limited. On soft sediments that are otherwise unsuitable for settlement, zebra mussel colonization creates expanding clusters of byssally-attached shells which act as preferred substrate for gregarious larvae (Berkman et al. 1998), whose conspecific attraction creates a positive feedback in local recruitment (Mortl and Rothhaupt 2003). The shells of other mollusks, particularly native unionid mussels, also serve as foci for initial settlement; attached zebra mussels add successively greater surface area to accelerate subsequent colonization (Ricciardi et al. 1995). Ultimately, the native mussel is smothered to death by the intense fouling, which impedes its normal feeding, respiration, excretion and valve movement. The level of mortality within the native unionid population is strongly correlated with the level of zebra mussel fouling on their shells, which in turn is dependent on the local population density of zebra mussels (Ricciardi et al. 1995, Ricciardi 2003). Total exclusion of unionid populations by zebra mussels can occur within 4-8 years of invasion (Ricciardi et al. 1995, 1996). In addition to their autogenic engineering activities, zebra mussels are highly efficient filter feeders that increase water clarity. The resultant change in transparency promotes the growth of weeds that act as substrate for settling mussel larvae, while species adapted to turbid water conditions (e.g. walleye, Sander vitreus) are excluded from the new habitat (Vanderploeg et al. 2002).

Unsuccessful invasions occur even when species are introduced into apparently suitable habitat (Fig. 4a); several failed introductions may precede the establishment of a selfsustaining population (Veltman et al. 1996). The hypothesized explanation for such events is lack of a sufficient number of introduced individuals to overcome demographic barriers (Fig. 4b-c; Lockwood et al. 2005). In cases where ecosystem engineering is necessary for establishment and its effects are density-dependent, it may contribute to the often observed time lag between the introduction and establishment of a species (Fig. 4b, 4d). Lag times are often on the order of decades (Crooks and Soule 1999, Crooks 2005). The Asian mussel Musculista senhousia was discovered on the Pacific coast of North America in the mid1960s, but did not become noticeably abundant until the early 1980s; by 1995, it achieved the highest densities $\left(170000 \mathrm{~m}^{-2}\right)$ ever recorded for the species (Crooks and Soulé 1999). A number of factors explain lag times, including physico-chemical factors, interspecific and intraspecific interactions, reduced reproductive success at low densities and the delayed selection of suitable genotypes (Shigesada and Kawasaki 1997, Crooks and Soulé 1999). The early lag phase of some animal invasions is explained by Allee effects, which are consistent with the non-linear case of our model. Lag times also precede the negative effects of ecosystem engineering; for example, intense grazing by introduced cattle in New Mexico suppressed the development of an entire age class of cottonwood trees over a 20 -year period, thereby reducing the trees available to act as food storage sites for birds, resulting in the dramatic decline of a once stable population of woodpeckers (Ligon and Stacey 1996).

The 'invasion collapse' scenario (Fig. 6d) could contribute to the phenomenon of dramatic population declines of exotic species, which are presumed to be caused by an unknown biotic and abiotic disturbance, competition with other introduced species, or boom-and-bust cycles, but usually without supporting evidence (Simberloff and Gibbons 2004). There are many cases in which an exotic species population is well established and, without management action, spontaneously crashes - sometimes to the point of extinction. Crashes may occur over large areas and involve plants as well as animals, but there is a paucity of examples involving ecosystem engineering species per se. After zebra mussels invade a lake they may experience irregular population fluctuations or a sudden crash (Ramcharan et al. 1992). Eurasian milfoil Myriophyllum spicatum, 
a highly invasive aquatic weed that engineers enemy-free space by producing phytotoxins to suppress competitors (Ervin and Wetzel 2003), has experienced rapid population crashes in many North American lakes; a variety of causes have been proposed, but none can explain the majority of cases (Jones et al. 1983, Creed 1998).

Finally, the legacy effect predicted by the model (in which a series of failed invasions results in an accumulation of engineered habitat that increases subsequent invasion success) might explain some of the mysterious cases of invasions succeeding after a long history of failed attempts, or after an extensive apparent lag time between initial introduction and establishment (Crooks 2005). For example, one might imagine a scenario in which a fouling bivalve succeeds in invading a muddy habitat after a sufficient amount of shell material is deposited by previous failed colonists (cf. Berkman et al. 1998).

\section{How persistent is the invader-transformed habitat?}

In both linear and non-linear cases the habitat transformation is reversible, but invasion success is enhanced by slower rates of degradation from the engineered state to the degraded state (Fig. 1). We also assumed that habitat degradation was density independent, thus reflecting an abiotic controlled process as opposed to a biotic controlled process (an assumption that requires further study). Empirical estimates of the rate at which the constructed habitat decays and is restored to its original state are scarce, in part because there have been relatively few successful exotic species eradications (Myers et al. 2000). The rate of restoration is highly variable among species. For allogenic engineers, the rate is usually a fraction of their generation time. Experiments have shown that only one year after the removal of introduced carp, turbidity is reduced by 40 $60 \%$ and native plants may grow in areas where they were previously excluded (Anderson 1950, Lougheed et al. 2004). Somewhat longer durations may occur for the restoration of altered chemical environments. The Mediterranean ice plant Mesembryanthemum crystallinum accumulates high concentrations of salts in its tissues, which are released into the surface soil after the plant dies and inhibit competition from native plants. After removal of the plant, the negative effects of augmented soil salinity persist for at least two years but decline over time (Vivrette and Muller 1977).

Decay and restoration rates are substantially lower for physical habitat constructed by autogenic engineers. Calcareous structures (shells, tubes, and exoskeletons) secreted by invertebrates (e.g. bivalves, corals, serpulid worms) can persist over large temporal and spatial scales where processes of mechanical and chemical erosion are negligible. Indeed, the effects of dead marine reef-building species may extend through geological time (Gutiérrez et al. 2003). In contrast, decay rates for zebra mussels and other freshwater bivalves are highly variable and are mediated by water chemistry and water movement; annual losses of bulk shell material may be $>99 \%$ in calcium-poor environments (Strayer and Malcom 2007). Thus, decay rates of habitat constructed by the same species can vary along environmental gradients.
The paucity of data on habitat degradation rates precludes more quantitative representation of this element of our model.

\section{Conclusions}

Feedbacks between invaders and invaded habitats further demonstrate the need for an integration of population biology and ecosystem ecology (Vitousek 1990). Progress toward this goal may be enabled by the ecosystem engineering concept, which allows the incorporation of dynamic interactions between species and abiotic environmental factors into ecological models (Odling-Smee et al. 2003, Cuddington et al. 2007, Hastings et al. 2007). Our model is a step in this direction. However, it omits a number of important aspects of natural systems. For example, we omitted evolutionary dynamics (Hänfling and Kollman 2002). Future analyses that explore the effect of contemporary evolution in models of ecosystem engineering will draw upon ideas central to the niche construction concept (e.g. environmentally mediated genetic effects, Odling-Smee et al. 2003). Here, we have described the ecological dynamics of invasion in which the invader modifies the habitat to the benefit of the invader and the detriment of native species. Future studies might also address how the presence of a resident engineer affects the invasion success of non-engineering or engineering exotic species.

Like the enemy release hypothesis (Keane and Crawley 2002, Colautti et al. 2004), ecosystem engineering explains why some invaders can dominate native species in environments to which the natives are presumably better adapted. It remains to be determined whether ecosystem engineering is broadly applicable to invasions, how often it is required for successful establishment, how often it results in the exclusion of resident species, how it varies among closelyrelated taxa, and whether highly successful or high-impact invaders are more likely to possess engineering traits. Another question of interest is whether exotic ecosystem engineering is more likely to benefit exotic species more than native species in the invaded environment. Ecosystem engineering could conceivably act as a biotic disturbance that enhances the success of resident exotic species by reducing antagonistic effects with previously-dominant natives (Hierro et al. 2006, Altman and Whitlatch 2007). Moreover, the success of introduced co-adapted species may be promoted by ecosystem engineering activities amongst a synergistic group, as has been observed among European feral pigs, plants and earthworms (Aplet et al. 1991, Mack and D'Antonio 1998). Clearly, further theoretical and empirical work is needed to develop a predictive understanding of the effects of exotic ecosystem engineering on biotic communities.

Acknowledgements - We thank Rebekah Kipp for commenting on the manuscript. Support from NSERC Canada (to AG and AR) and a Canada Research Chair in Biodiversity (to AG) is gratefully acknowledged. AL acknowledges the ANR (French national 
research agency) for funding (project stochastic models for the evolution of life).

\section{References}

Altman, S. and Whitlatch, R. B. 2007. Effects of small-scale disturbance on invasion success in marine communities. - J. Exp. Mar. Biol. Ecol. 342: 15-29.

Anderson, J. M. 1950. Some aquatic vegetation changes following fish removal. - J. Wildlife Manage. 14: 206-209.

Aplet, G. H. et al. 1991 Association between feral pig disturbance and the composition of some alien plant assemblages in Hawaii Volcanoes National Park. - Vegetatio 95: 55-62.

Bais, H. P. et al. 2003. Allelopathy and exotic plant invasion: from molecules and genes to species interactions. - Science 301: 1377-1380.

Berkman, P. A. et al. 1998. Zebra mussels invade Lake Erie muds. - Nature 393: 27-28.

Brooks, M. L. et al. 2004 Effects of invasive alien plants on fire regimes. - BioScience 54: 677-688.

Callaway, R. M. et al. 2004. Soil biota and exotic plant invasion. - Nature 427: 731-733.

Colautti, R. I. et al. 2004. Is invasion success explained by the enemy release hypothesis? - Ecol. Lett. 7: 721-733.

Creed, R. P. 1998. A biogeographic perspective on Eurasian milfoil declines: additional evidence for the role of herbivorous weevils in promoting declines? - J. Aquat. Plant Manage. 36: 16-22.

Crooks, J. A. 2002. Characterizing ecosystem-level consequences of biological invasions: the role of ecosystem engineers. - Oikos 97: 153-166.

Crooks, J. A. 2005. Lag times and exotic species: the ecology and management of biological invasions in slow-motion. - Ecoscience 12: 316-329.

Crooks, J. A. and Soulé, M. E. 1999. Lag times in population explosions of invasive species: causes and implications. - In: Sandlund, O. T. et al. (eds), Invasive species and biodiversity management. Kluwer, pp. 103-125.

Cuddington, K. and Hastings, A. 2004. Invasive engineers. - Ecol. Modell. 178: 335-347.

Cuddington, K. et al. 2007. Ecosystem engineers. - Academic Press.

D'Antonio, C. M. and Vitousek, P. M. 1992. Biological invasions by exotic grasses, the grass/fire cycle, and global change. - Annu. Rev. Ecol. Syst. 23: 63-87.

Davis, M. A. et al. 2000. Fluctuating resources in plant communities: a general theory of invasibility. - J. Ecol. 88: 528-534.

Duda, J. J. et al. 2003. Differences in native soil ecology associated with invasion of the exotic annual chenopod, Halogeton glomeratus. - Biol. Fertility Soils 38: 72-77.

Ervin, G. N. and Wetzel, R. G. 2003. An ecological perspective of allelochemical interference in land-water interface communities. - Plant Soil 256: 13-28.

Gido, K. B. and Brown, J. H. 1999. Invasion of North American drainages by alien fish species. - Freshwater Biol. 42: 387-399.

Gurney, W. S. C. and Lawton, J. H. 1996. The population dynamics of ecosystem engineers. - Oikos 76: 273-283.

Gutiérrez, J. L. et al. 2003. Mollusks as ecosystem engineers: the role of shell production in aquatic habitats. - Oikos 101: 7990.

Hastings, A. et al. 2007. Ecosystem engineering in space and time. - Ecol. Lett. 10: 153-164.

Hänfling, B. and Kollman, J. 2002. An evolutionary perspective of biological invasions. - Trends Ecol. Evol. 17: 545-546.
Hierro, J. L. et al. 2006. Disturbance facilitates invasion: the effects are stronger abroad than at home. - Am. Nat. 167: $144-156$.

Jones, C. G. et al. 1997. Positive and negative effects of organisms as physical ecosystem engineers. - Ecology 78: 1946-1957.

Jones R. C. et al. 1983. Phytoplankton as a factor in the decline of the submersed macrophyte Myriophyllum spicatum L. in Lake Wingra, Wisconsin, USA. - Hydrobiologia 107: 213-219.

Keane, R. M. and Crawley, M. J. 2002. Exotic plant invasions and the enemy release hypothesis. - Trends Ecol. Evol. 17: 164170.

Kourtev, P. S. et al. 2002. Exotic plant species alter the microbial community structure and function in the soil. - Ecology 83: 3152-3166.

Ligon, J. D. and Stacey, P. B. 1996. Land use, lag times and the detection of demographic change: the case of the acorn woodpecker. - Conserv. Biol. 10: 840-846.

Link, S. O. et al. 2006 Bromus tectorum cover mapping and fire risk. - Int. J. Wildland Fire 15: 113-119.

Lockwood, J. L. et al. 2005. The role of propagule pressure in explaining species invasions. - Trends Ecol. Evol. 20: 223228.

Lougheed, V. L. et al. 1998. Predictions on the effect of common carp (Cyprinus carpio) exclusion on water quality, zooplankton, and submergent macrophytes in a Great Lakes wetland. - Can. J. Fish. Aquat. Sci. 55: 1189-1197.

Lougheed, V. L. et al. 2004. Carp exclusion, food-web interactions and the restoration of Cootes Paradise Marsh. - J. Great Lakes Res. 30: 44-57.

Mack, M. C. and D’Antonio, C. M. 1998 Impacts of biological invasions on disturbance regimes. - Trends Ecol. Evol. 13: 195-198.

Mortl, M. and Rothhaupt, K. O. 2003. Effects of adult Dreissena polymorpha on settling juveniles and associated macroinvertebrates. - Int. J. Hydrobiol. 88: 561-569.

Myers, J. H. et al. 2000. Eradication revisited: dealing with exotic species. - Trends Ecol. Evol. 17: 316-320.

Ntiba, M. J. et al. 2001. Management issues in the Lake Victoria watershed. - Lake Reserv. Manage. 6: 211-216.

Odling-Smee, F. J. et al. 2003. Niche construction: the neglected process in evolution. - Princeton Univ. Press.

Pipalova, I. 2002. Initial impact of low stocking density of grass carp on aquatic macrophytes. - Aquat. Bot. 73: 9-18.

Ramcharan, C. W. et al. 1992. A multivariate model for predicting population fluctuations of Dreissena polymorpha in North American lakes. - Can. J. Fish. Aquat. Sci. 49: 150158.

Ricciardi, A. 2003. Predicting the impacts of an introduced species from its invasion history: an empirical approach applied to zebra mussel invasions. - Freshwater Biol. 48: 972-981.

Ricciardi, A. et al. 1995. Predicting the intensity and impact of Dreissena infestation on native unionid bivalves from Dreissena field density. - Can. J. Fish. Aquat. Sci. 52: 1449-1461.

Ricciardi, A. et al. 1996. Impact of the Dreissena invasion on native unionid bivalves in the upper St. Lawrence River. - Can. J. Fish. Aquat. Sci. 53: 1434-1444.

Richardson, D. M. et al. 2000. Naturalization and invasion of alien plants: concepts and definitions. - Div. Distr. 6: 93-107.

Richardson, M. J. 1996. Factors limiting the colonization success of an introduced exotic fish (Carassius auratus). $\mathrm{PhD}$ thesis. - McGill Univ., Montreal.

Richardson, M. J. and Whoriskey, F. G. 1992. Factors influencing the production of turbidity by goldfish (Carassius auratus). - Can. J. Zool. 70: 1585-1589.

Richardson, M. J. et al. 1995. Turbidity generation and biological impacts of an exotic fish Carassius auratus, introduced into shallow seasonally anoxic ponds. - J. Fish Biol. 47: 576-585. 
Sax, D. F. and Brown, J. H. 2000. The paradox of invasion. - Global Ecol. Biogeogr. 9: 363-371.

Sax, D. F. et al. 2002. Species invasions exceed extinctions on islands worldwide: a comparative study of plants and birds. - Am. Nat. 160: 766-783.

Scheffer, M. et al. 2003. Floating plant dominance as a stable state. - Proc. Natl Acad. Sci. USA 100: 4040-4045.

Shigesada, N. and Kawasaki, K. 1997. Biological invasions: theory and practice. - Oxford Univ. Press.

Simberloff, D. and Gibbons, L. 2004. Now you see them, now you don't! - population crashes of established introduced species. - Biol. Invas. 6: 161-172.

Strayer, D. L. and Malcom, H. M. 2007. Shell decay rates of native and alien freshwater bivalves and implications for habitat engineering. - Freshwater Biol. 52: 1611-1617.

Talley, T. S. and Levin, L. A. 2001. Modification of sediments and macrofauna by an invasive marsh plant. - Biol. Invas. 3: 5168.

Taylor, C. M. and Hastings, A. 2005. Allee effects in biological invasions. - Ecol. Lett. 8: 895-908.

van den Brink, F. W. B. et al. 1993. Ecological aspects, explosive range extension and impact of a mass invader, Corophium curvispinum Sars, 1895 (Crustacea: Amphipoda), in the lower Rhine (the Netherlands). - Oecologia 93: 224-232.

van der Velde, G. et al. 1994. Decline of zebra mussel populations in the Rhine: competition between two mass invaders
(Dreissena polymorpha and Corophium curvispinum). - Naturwissenschaften 81: 32-34.

Vanderploeg, H. A. et al. 2002. Dispersal and emerging ecological impacts of Pont-Caspian species in the Laurentian Great Lakes. - Can. J. Fish. Aquat. Sci. 59: 1209-1228.

Veltman, C. J. et al. 1996. Correlates of introduction success in exotic New Zealand birds. - Am. Nat. 147: 542-557.

Vilá, M. and Weiner, J. 2004. Are invasive plant species better competitors than native plant species - evidence from pairwise experiments. - Oikos 105: 229-238.

Vitousek, P. M. 1990. Biological invasions and ecosystem processes: towards an integration of population biology and ecosystem studies. - Oikos 57: 7-13.

Vitousek, P. M. et al. 1997. Introduced species: a significant component of human-caused global change. - N. Z. J. Ecol. 21: $1-16$.

Vivrette, N. J. and Muller, C. H. 1977. Mechanism of invasion and dominance of coastal grassland by Mesembryanthemum crystallinum. - Ecol. Monogr. 47: 301-318.

Yang, S. Y. et al. 1992. Isolation and identification of antialgal compounds in root system of water hyacinth. - Acta Phytophysiol. Sin. 18: 399-402.

Williamson, M. 1996. Biological invasions. - Chapman and Hall.

Zambrano, L. et al. 1999. Impact of introduced carp (Cyprinus carpio) in subtropical shallow ponds in Central Mexico. - J. Aquat. Ecosyst. Stress Recovery 6: 281-288. 